\title{
Hepatitis B virus suppression predicts better recurrence-free survivals in liver transplant patients with hepatocellular carcinoma
}

Su Young Hong, Kwang-Woong Lee, Sola Lee, Sanggyun Suh, Eui Soo Han, Suk Kyun Hong, YoungRok Choi, Nam-Joon Yi, Kyung-Suk Suh

Department of Surgery, Seoul National University College of Medicine, Seoul, Korea

Background: High serum load of hepatitis B virus (HBV) deoxyribonucleic acid (DNA) is known to be a strong risk factor of hepatocellular carcinoma (HCC) development. The aim of study was to investigate the predictive role of HBV DNA levels in recurrence of HCC after liver transplantation (LT).

Methods: From June 2006 to May 2020, 729 recipients underwent LT for HBV-related HCC in Seoul National University Hospital. The risk factors for HCC recurrence after LT were analyzed including serum HBV DNA load.

Results: Recurrence-free survival at 1, 3, 5, and 10 years were $99.6 \%, 98 \%, 95.1 \%$, and $87.8 \%$, respectively. Detectable HBV DNA level (higher than $10 \mathrm{IU} / \mathrm{mL}$ ) before transplant was significant predictors of $\mathrm{HCC}$ recurrence in univariate analysis $(P=0.027)$. Further subgroup analysis was performed to demonstrate the significance of HBV DNA level according to the risk of HCC recurrence. Based on the score of the predicted survival after LT for HCC (SALT), patients were divided in three groups. In high-risk group of recurrence with SALT score more than 2.44, detectable HBV DNA level were significantly associated with recurrence free survival $(57.9 \%$ vs. $78.7 \%, \mathrm{P}<0.0001)$.

Conclusions: There is a close relationship between HBV DNA level and HCC recurrence after transplant. High HBV DNA levels before transplant are associated with HCC recurrence after transplant, especially in high recurrence risk group. 